\title{
Microfabrication of polystyrene microbead arrays by laser induced forward transfer
}

\author{
Alexandra Palla-Papavlu, ${ }^{1, a)}$ Valentina Dinca, ${ }^{1}$ lurie Paraico, ${ }^{2}$ Antoniu Moldovan, ${ }^{1}$ \\ James Shaw-Stewart, ${ }^{3}$ Christof W. Schneider, ${ }^{4}$ Eugenia Kovacs, ${ }^{2}$ Thomas Lippert, ${ }^{4}$ and \\ Maria Dinescu ${ }^{1}$ \\ ${ }^{1}$ National Institute for Lasers, Plasma and Radiation Physics, P.O. Box MG-36, Magurele RO-077125, \\ Bucharest, Romania \\ ${ }^{2}$ Department of Biophysics and Cell Biotechnology, Carol Davila University of Medicine and Pharmacy, \\ P.O. Box 35-43, 50474, Bucharest, Romania \\ ${ }^{3}$ Laboratory for Functional Polymers, Swiss Federal Laboratories for Materials Testing and Research, \\ EMPA, Überlandstrasse 129, 8600 Dübendorf, Switzerland \\ ${ }^{4}$ Department of General Energy Research, Paul Scherrer Institute, 5232 Villigen PSI, Switzerland
}

(Received 20 April 2010; accepted 16 June 2010; published online 10 August 2010)

\begin{abstract}
In this study we describe a simple method to fabricate microarrays of polystyrene microbeads (PS- $\mu$ beads) on Thermanox coverslip surfaces using laser induced forward transfer (LIFT). A triazene polymer layer which acts as a dynamic release layer and propels the closely packed microspheres on the receiving substrate was used for this approach. The deposited features were characterized by optical microscopy, scanning electron microscopy, atomic force microscopy, and Raman spectroscopy. Ultrasonication was used to test the adherence of the transferred beads. In addition, the laser ejection of the PS- $\mu$ bead pixels was investigated by time resolved shadowgraphy. It was found that stable PS- $\mu$ beads micropatterns without any specific immobilization process could be realized by LIFT. These results highlight the increasing role of LIFT in the development of biomaterials, drug delivery, and tissue engineering. (C) 2010 American Institute of Physics. [doi:10.1063/1.3466746]
\end{abstract}

\section{INTRODUCTION}

The simultaneous development of biophysics, biochemistry, and semiconductor physics results in the creation of a new class of devices i.e., biochips. Their use in life sciences is spreading more and more in applications such as molecular diagnostics, ${ }^{1}$ proteomics, ${ }^{2}$ and genomics. ${ }^{3}$ In that context, polystyrene microbead (PS- $\mu$ bead) microarrays represent a promising approach as a low cost solution for collecting independently data from numerous beads in order to provide statistical rigor, which is useful for applications including biosensing, bioseparation, and biomolecule screening. PS- $\mu$ bead arrays have been fabricated by different techniques, i.e., hydrophobic interactions, ${ }^{4}$ through the use of porous films, ${ }^{5}$ crosslinking, ${ }^{6}$ dielectrophoretics, ${ }^{7}$ pin microspotting, ${ }^{8}$ and inkjet printing. ${ }^{9}$ The overall disadvantage of these methods is the lack of variability in the design of the pattern and in the adherence of the patterned microbeads onto various surfaces. ${ }^{10}$

In order to overcome these difficulties an attractive alternative that avoids the use of expensive photolithographic masks and presents higher integration scale than microspotting or ink-jet printing could be laser induced forward transfer (LIFT). ${ }^{11}$ LIFT is a versatile technique which has been used for printing patterns of different materials, including metals, ${ }^{12}$ oxides, ${ }^{13}$ nanoparticles, ${ }^{14}$ polymers, and biomolecules, i.e., proteins, ${ }^{15,16}$ DNA, ${ }^{17,18}$ cells, ${ }^{19,20}$ and tissue. ${ }^{21}$

In LIFT the material is transferred by the laser beam from a transparent support or donor onto an appropriate sub-

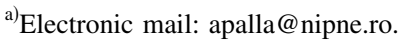

strate or receiver. For improving the process efficiency and reducing the risk of damaging the layer to be transferred, the donor substrate can be previously coated with a polymeric layer, which is called dynamic release layer (DRL) or sacrificial layer. ${ }^{22,23}$

In our approach, the polymeric layer used as DRL was a triazene polymer (TP) ${ }^{24}$ specifically designed for an irradiation wavelength of $308 \mathrm{~nm}$. This triazene layer absorbs the laser radiation, decomposes into small, gaseous, molecular fragments and propels the material to be transferred. The use of a triazene polymer as DRL has numerous advantages, i.e., (i) the triazene polymer has a very low ablation threshold of $25 \mathrm{~mJ} / \mathrm{cm}^{2}$ for $308 \mathrm{~nm}$ irradiation which means that the transfer can be achieved with low thermal impact on the donor layer; (ii) no debris is detected, and (iii) a high absorption coefficient at the $\mathrm{XeCl}$ excimer laser wavelength. ${ }^{25-27}$

The aim of this study is to establish the optimum parameters employed for obtaining stable PS- $\mu$ beads micropatterns without any specific immobilization process.

\section{MATERIALS AND METHODS}

\section{A. Donor structures}

The donor substrates were prepared by spin coating the triazene polymer (with a thickness of around $100 \mathrm{~nm}$ ) onto fused silica plates (area $5 \mathrm{~cm}^{2}$, thickness $1 \mathrm{~mm}$ ) and then drop casting the PS- $\mu$ beads (size: $8 \mu \mathrm{m}$ diameter from Sigma Aldrich, No. 78511) on top of the triazene polymer layer. Due to the deposition method of the PS- $\mu$ beads the areas of close-packed monolayers were, typically, of the or- 


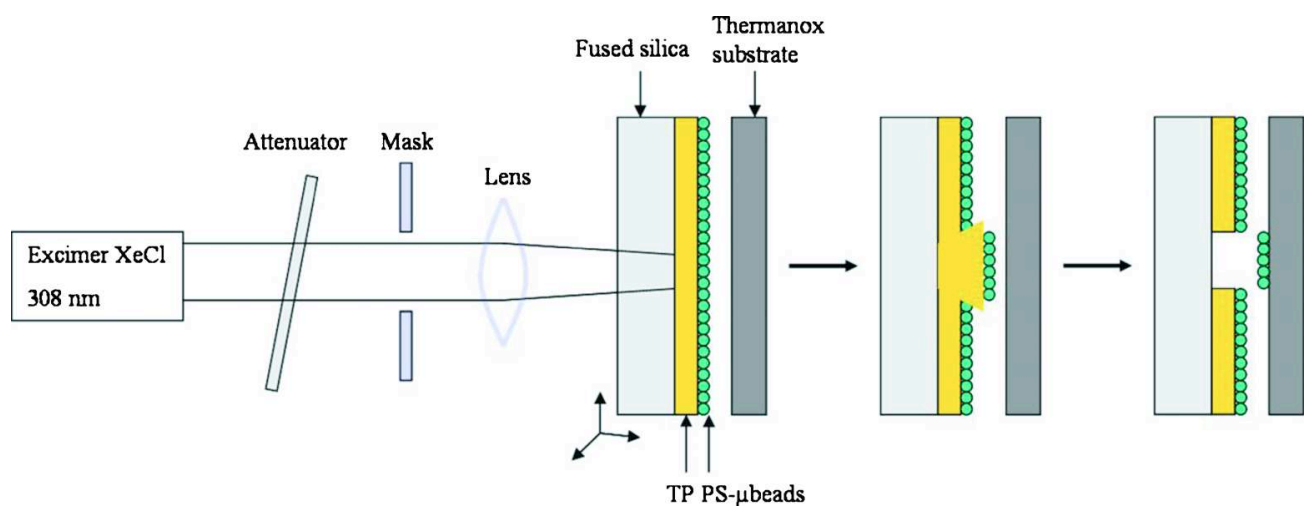

FIG. 1. (Color online) Scheme of the DRL-assisted LIFT process.

der of approximately tens of micron square. The TP was synthesized as described by Nagel et al. in Ref. 24 and was then prepared by spin coating from a solution in chlorobenzene and cyclohexanone $(1: 1, \mathrm{w} / \mathrm{w})$.

\section{B. Receiver substrates}

The receiver substrates were round Thermanox coverslips with a diameter of $13 \mathrm{~mm}$. Thermanox coverslips were chosen as substrates due to their wide applications and properties, i.e., high resistance to most chemicals, enhanced cell attachment and growth, and a lower hardness as compared to glass slides or fused silica plates.

\section{Patterning system}

The scheme of the DRL-assisted LIFT principle is presented in Fig. 1. A XeCl excimer laser with an emission wavelength of $308 \mathrm{~nm}$ (Lambda Physik, $30 \mathrm{~ns}$ pulse length) was used for the transfer. The laser fluence was varied in the range of $80 \mathrm{~mJ} / \mathrm{cm}^{2}-3.5 \mathrm{~J} / \mathrm{cm}^{2}$. The donor and the receiver were placed in closed contact on a computer controlled translation stage while the laser irradiates the donor from the backside. The laser beam passed through a mask with an adjustable aperture and was focused by a lens to a spot size of $200 \times 200 \mu \mathrm{m}^{2}$. For each laser pulse PS- $\mu$ beads pixels were obtained which were arranged in a matrix of points.

\section{Morphological and chemical characterization of the deposits}

The deposited features as well as the target prior to ablation were investigated by optical microscopy. The images were acquired with an Axiovert 200 Microscope coupled to a Carl Zeiss AxioCam MRm camera. The distribution of the PS $\mu$ spheres on the receiving surface was investigated by a scanning electron microscope (SEM) operating at a voltage of $5 \mathrm{kV}$ and an in-lens detector. The samples were coated with a $3 \mathrm{~nm}$ thin Pt film for conductivity. Atomic force microscopy (AFM) images of the morphology of the transferred pixels were acquired with a XE 100 System from Park.

Raman spectroscopy was used to check for any chemical changes in the structure of the PS $\mu$ spheres after their transfer. The Raman spectra of PS- $\mu$ beads transferred at different fluences were recorded on a Labram HR or Labram confocal
Raman microscopy system from Jobin Yvon. The $633 \mathrm{~nm}$ line from a HeNe laser was used as an excitation source for the Raman spectra. The laser power at the sample surface was typically $20 \mathrm{~mW}$. For each measurement, the exposure time and the accumulation were $20 \mathrm{~s}$ and five times, respectively. Raman spectra were collected over the range of $300-3300 \mathrm{~cm}^{-1}$. All spectra were recorded at room temperature but also at different temperatures using a Linkam heating stage.

\section{E. Shadowgraphy imaging setup}

In order to perform the nanosecond-shadowgraphy experiments, a camera with a zoom objective and the dye used for illumination were positioned parallel to the sample surface. The illumination was obtained with a Nd:YAG (from Quantel Brilliant B, $5 \mathrm{~ns}$ pulse length) laser operating at the second harmonic (532 $\mathrm{nm}$ wavelength) that was pumping a fluorescent dye (Rhodamine) ${ }^{28}$ All measurements were performed under ambient pressure at room temperature.

\section{F. Adherence tests}

To test the adherence of the PS- $\mu$ beads pixels onto the Thermanox coverslip substrate, sonication was performed in a focused ultrasonic bath (Bandelin Sonopuls HD-2070), with a processing frequency of $20 \mathrm{kHz}$ and uniform sonic waves. For this test each sample was immersed in $10 \mathrm{ml}$ double distilled water in a falcon tube. The experimental parameters applied for testing the adherence of the PS- $\mu$ bead pixels on the Thermanox coverslips substrates are listed in Table I.

\section{RESULTS AND DISCUSSION}

\section{A. Influence of the laser fluence on the transferred pixels}

Optical micrographs of PS- $\mu$ bead pixels obtained immediately after transfer at different fluences are displayed in Fig. 2. The laser fluence was varied over a broad range, i.e., from conditions insufficient to break the donor layer to a high irradiation fluences $\left(0.08-3.5 \mathrm{~J} / \mathrm{cm}^{2}\right)$, in order to optimize the shape of the transferred pixels without any chemical modification of the PS-beads. The threshold fluence for the complete removal and transfer of the PS- $\mu$ bead thin films 
TABLE I. Operating program 1 means that in $1 \mathrm{~s}$ for $0.1 \mathrm{~s}$ a sonication pulse of $20 \mathrm{kHz}$ is emitted. Operating program 9 (represents the maximum value of the pulse mode) means that for 0.9 seconds a pulse is emitted. Each operating program was repeated ten times. Continuous operating program means that the sonication frequency is emitted permanently for the entire second.

\begin{tabular}{cccc}
\hline \hline Time & $\begin{array}{c}\text { Power } \\
(\%)\end{array}$ & Operating program $(10 \times)$ & Mode \\
\hline $3 \mathrm{~s}$ & 50 & 1 & Pulse \\
$10 \mathrm{~s}$ & 50 & 1 & Pulse \\
$30 \mathrm{~s}$ & 50 & 9 & Pulse \\
$30 \mathrm{~s}$ & 100 & 9 & Pulse \\
$1 \mathrm{~min}$ & 100 & 9 & Pulse \\
$5 \mathrm{~min}$ & 50 & 9 & Pulse \\
$5 \mathrm{~min}$ & 100 & Continuous & Constant \\
$10 \mathrm{~min}$ & 100 & Continuous & Constant \\
\hline \hline
\end{tabular}

from the irradiated area (a $200 \times 200 \mu \mathrm{m}^{2}$ spot) is $0.71 \mathrm{~J} / \mathrm{cm}^{2}$, which is much higher than the fluence needed for the complete delamination of a triazene polymer layer with the same thickness $(100 \mathrm{~nm})$, which occurs at a fluence of $35 \mathrm{~mJ} / \mathrm{cm}^{2} .^{23}$ The fluence necessary for the transfer of the PS-beads $\left(>710 \mathrm{~mJ} / \mathrm{cm}^{2}\right)$ will therefore completely decompose the TP layer into gaseous products, because the necessary fluence for the ablation of a $100 \mathrm{~nm}$ TP layer is around $50 \mathrm{~mJ} / \mathrm{cm}^{2} .^{23}$ It is therefore very probable that no contamination of the deposited PS- $\mu$ beads with debris from the ablation of the TP will be observed. Below $0.71 \mathrm{~J} / \mathrm{cm}^{2}$ only single beads are transferred [see Fig. 2] while for fluences up to $3.5 \mathrm{~J} / \mathrm{cm}^{2}$ no damage of the PS- $\mu$ beads is detected.

The transfer of PS- $\mu$ beads without a release layer and all applied conditions results only in the transfer of randomly distributed beads, i.e., never a bead array, on the receiver substrate, which confirms the importance of the DRL.

Further investigations are necessary to investigate the correlation between the thickness of the triazene polymer layer and PS- $\mu$ bead monolayer.

\section{B. Morphological and chemical characterization of PS- $\mu$ bead pixels}

For the fabrication of a microanalytical device it is very important to control both the size and the shape of the transferred spot.
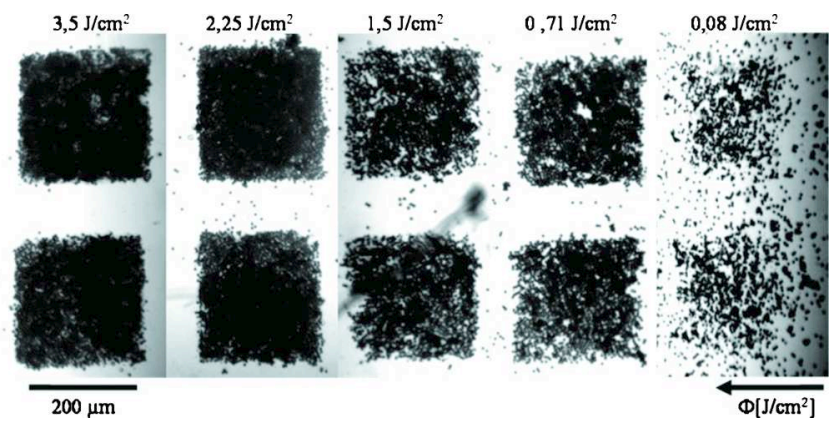

FIG. 2. (Color online) Optical micrographs of PS- $\mu$ bead pixels obtained immediately after transfer. The area of the pixels is $200 \times 200 \mu \mathrm{m}^{2}$. The laser fluences applied for the transfer process increase from right to left.

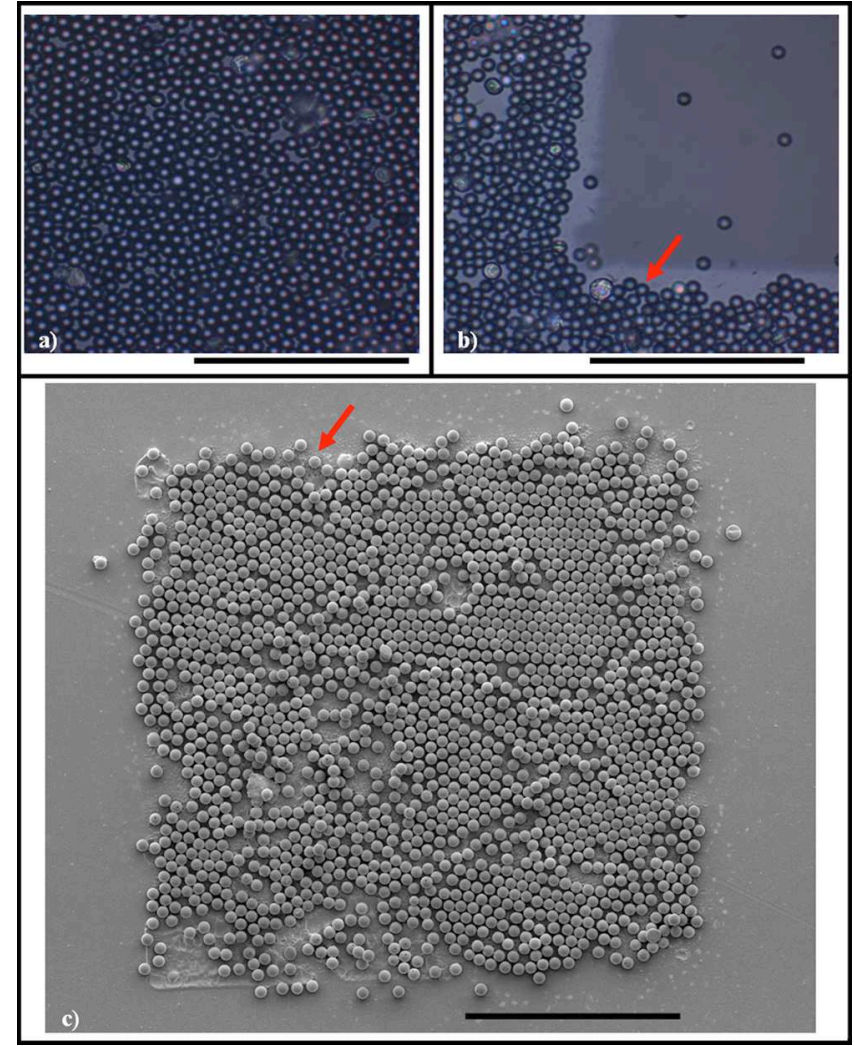

FIG. 3. (Color online) Optical image of a $\sim 0.4 \mathrm{~mm}^{2}$ area of (a) PS- $\mu$ beads monolayer from the target prior to ablation and (b) area of the target immediately after laser transfer. (c) SEM image of a pixel printed with a laser fluence of $2.25 \mathrm{~J} / \mathrm{cm}^{2}$. The scale bar corresponds to $100 \mu \mathrm{m}$.

In Fig. 2 micro-PS bead pixels ( 2 experiments) transferred with different laser fluences are shown. All the pixels present a similar size of about $(200 \times 200) \mu \mathrm{m}^{2}$ but in some cases the edges are less uniform, e.g., the left bottom corner at $1.5 \mathrm{~J} / \mathrm{cm}$. This is probably due to inhomogeneities of the DRL film thickness or to pulse to pulse energy variation in the laser. All transferred pixels are regularly arranged with some single beads deposited between the arrays. These beads can be easily removed by a soft nitrogen stream, which leaves the arrays intact.

A typical area of closely packed PS- $\mu$ beads from the target (donor substrate) before irradiation is shown in Fig. 3(a). When a laser pulse is applied, the PS- $\mu$ beads are completely detached from the target leaving a clean surface [Fig. 3(b)]. A SEM image of a transferred pixel is shown in Fig. $3(\mathrm{c})$. The features noticed at the edges of this pixel correspond well to the features observed in the target after laser irradiation. The nonhomogeneities which are present in the pixel, i.e., randomly distributed holes are due to the fact that the areas of close-packed PS- $\mu$ beads are only of the order of approximately tens of micron square. A SEM image with higher magnification of a pixel (shown in Fig. 4) reveals that the $8 \mu \mathrm{m}$ PS spheres are immobilized on the Thermanox substrate. The transferred microbeads have a clean surface with no visible changes in the morphology or debris from the triazene polymer. The AFM images of the PS- $\mu$ bead transferred on Thermanox coverslips surface are consistent with the SEM results (Fig. 4). A topographic AFM image of the 


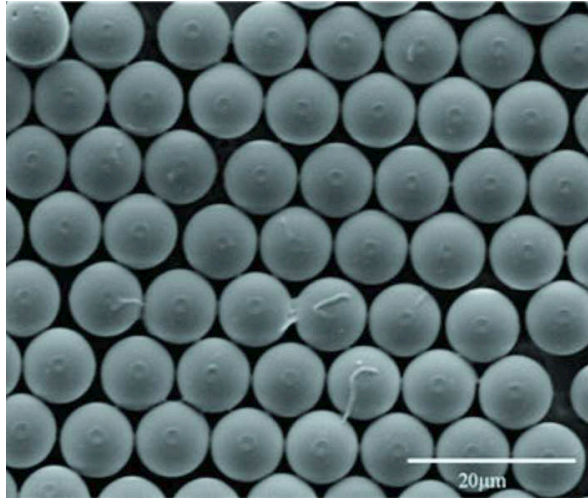

FIG. 4. (Color online) SEM micrograph of the surface morphology of the PS- $\mu$ bead transferred by LIFT at $1.5 \mathrm{~J} / \mathrm{cm}^{2}$.

surface of a PS- $\mu$ bead microarray is shown in Fig. 5, confirming the perfect alignment of the beads and their debris free surface.

A question difficult to address is the influence of a potential heat transfer during the ablation process thereby potentially affecting the structural and chemical integrity of the PS- $\mu$ beads. Using $\mu$-Raman offers the possibility to investigate the chemical composition of thin films or layers even with rough surfaces. The $\mu$-Raman reference spectrum [Fig. $6(\mathrm{a})$, bottom] of the beads agrees well with literature data ${ }^{29}$ and the assignment of the most important peaks is summarized in Table II. Raman spectra taken of transferred PS $\mu$-beads using different laser fluences [see Fig. 6(a)] show a clear tendency: the intensity of characteristic Raman peaks decreases with increasing laser fluence or the intensity ratio between neighboring peaks changes in a characteristic way. This is most prominent for the two bands around $999 \mathrm{~cm}^{-1}$ where the intensity ratio for the two peaks changes from approximately $2: 1$ for the reference sample to $3: 1$ for the highest fluence. Still, the Raman spectra taken at different fluences can be identified as polystyrene, suggesting that no chemical modification of the beads takes place upon laser transfer. In addition, there are no indications of triazene being present in the transferred $\mu$-beads. Several films consist-

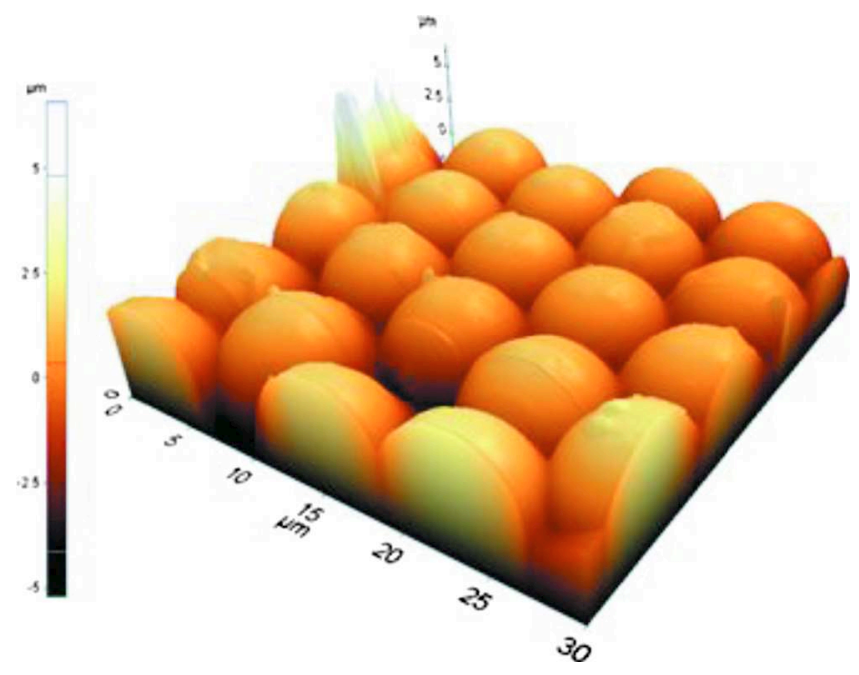

FIG. 5. (Color online) AFM image of PS- $\mu$ bead deposited on a Thermanox coverslip at $0.71 \mathrm{~J} / \mathrm{cm}^{2}$. ing of PS $\mu$-beads have been prepared and measured every $10{ }^{\circ} \mathrm{C}$ between room temperature and the melting temperature of polystyrene $\left(T=240{ }^{\circ} \mathrm{C}\right)$ using Raman microscopy [see Fig. 6(b)] to test whether the observed changes, i.e., decrease in intensity with increasing transfer fluence, are an indication of a heat transfer during the ablation process. The observed changes in intensity and intensity ratios are, up to 200-210 ${ }^{\circ} \mathrm{C}$, in the spectra small and comparable to changes shown in Fig. 6(a). Reaching $230^{\circ} \mathrm{C}$, rapid changes in the spectrum take place and at the melting temperature, only some rudimentary bands of the original polystyrene are left. These observed changes near and above the PS melting temperature are irreversible, i.e., even after cooling to room temperature no typical polystyrene spectrum was obtained. From these temperature dependent measurements we conclude, that the transferred PS $\mu$-beads are subjected to a minor temperature rise of at most $200{ }^{\circ} \mathrm{C}$ while ablating a pixel with the highest laser fluence of $3.5 \mathrm{~J} / \mathrm{cm}^{2}$. The possible origin of a heat transfer is the thermal decomposition process of the triazene caused by the laser radiation or even a direct intake of the transferred laser energy.

\section{Shadowgraphy imaging}

For the visualization of the laser transfer process time resolved shadowgraphy (scheme shown in Fig. 7) was applied which allows the visualization of the shockwave propagation and of the moving material in the initial stages of the LIFT process. A sequence of pictures taken for the LIFT process of a PS- $\mu$ bead monolayer on top of a $100 \mathrm{~nm}$ triazene polymer film for an irradiation fluence of $1.5 \mathrm{~J} / \mathrm{cm}^{2}$ is presented in Fig. 8. The laser irradiates the donor from the back side, i.e., from the right in Fig. 8. The absorption of the laser beam takes place in the triazene layer which is vaporized and a large amount of gas is released during the laser pulse. $^{28}$

The shockwave and its expansion are clearly visible, i.e., as a dark semicircle after $200 \mathrm{~ns}$. Material movement of the beads starts for single beads around $200 \mathrm{~ns}$, while the movement of a complete bead layer is observed after 400-600 ns, with a clear separation of the layer from the substrate after 1600 ns. The layer appears clearly thicker than the thickness of the $8 \mu \mathrm{m}$ bead layer, which is either due to a nonperfect perpendicular alignment of the camera axis with the flyer or due to nondense bead layer which expands upon movement. The flyer is highly forward directed and keeps its cohesion for distances of about $50 \mu \mathrm{m}$, which is consistent with the observation that for larger distances the PS bead pixel array did not reach the receiver surface.

For low fluences $<0.08 \mathrm{~J} / \mathrm{cm}^{2}$ a well-defined delamination of the flyer is not observed while for all higher fluences well-defined flyers are obtained. Both observations correspond quite well to the fluence onset and fluence range for the array transfer shown in Fig. 2.

The nanosecond-shadowgraphy experiments suggest strongly that the pixels remained intact during the flight for fluences $>700 \mathrm{~mJ} / \mathrm{cm}^{-2}$ and that array is transferred as an "intact" layer. 

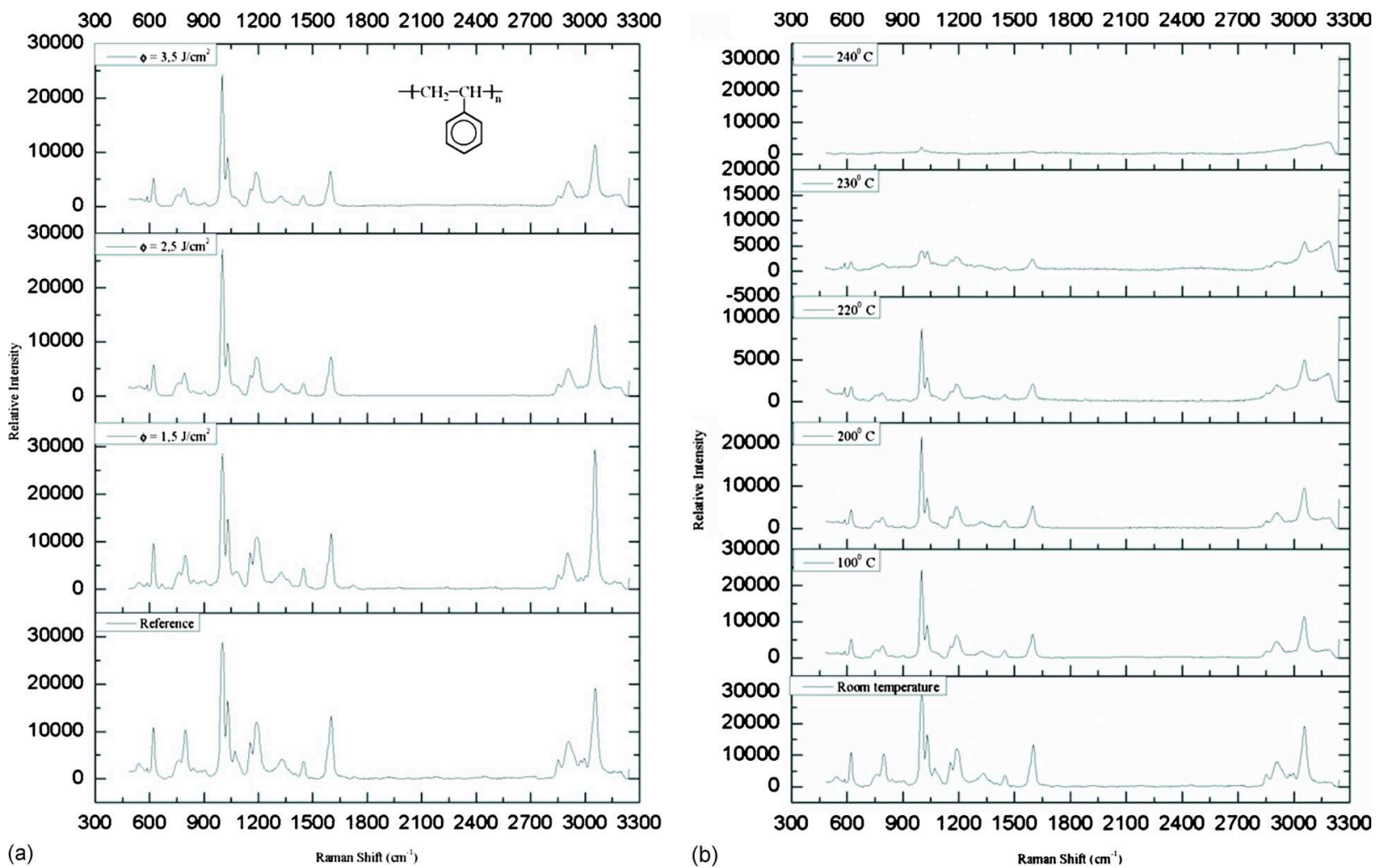

FIG. 6. (Color online) (a) Typical Raman spectra of PS-beads as acquired in the spectral region $300-3300 \mathrm{~cm}^{-1}$ (b) Raman spectra of PS-beads as a function of temperature.

\section{Adherence tests}

The transferred PS- $\mu$ bead pixels must withstand harsh conditions when applied in various biological environments, i.e., $p \mathrm{H}$, controlled flow, and changes in temperature, ionic strength. ${ }^{30}$

The PS- $\mu$ beads transferred by LIFT are very adherent to the surface of the substrate. Although the PS- $\mu$ beads could easily be scratched off mechanically, they stayed intact after ultrasonicating in double distilled water for up to $10 \mathrm{~min}$. The good adherence of the laser transferred pixels may be due to the strong/fast impact of the PS-beads on the substrates. This strong impact is due to the pressure jump resulting from the decomposition of the triazene polymer layer into gaseous fragments, which may reach several gigapascal. ${ }^{31}$ Our results highlight that LIFT is a promising time- and cost-effective technique for obtaining firmly attached PS-beads to Thermanox substrates compared to other techniques that require complicated protocols ${ }^{32}$ for obtaining PS- $\mu$ beads microarrays.

TABLE II. Vibrational assignment of the most important Raman bands of PS- $\mu$ beads.

\begin{tabular}{cc}
\hline \hline $\begin{array}{c}\text { Bands } \\
\left(\mathrm{cm}^{-1}\right)\end{array}$ & Assignment \\
\hline 999 & Symmetrical benzene ring breathing frequency \\
1031 & Hydrogen bending mode \\
1449 & Bending mode, $\delta\left(\mathrm{CH}_{2}\right)$ \\
1606 & Ring frequency \\
3056 & Hydrogen stretching \\
3066 & Benzene modes \\
\hline \hline
\end{tabular}

\section{CONCLUSION}

We show in this work that the use of laser processing techniques, i.e., LIFT, is a promising approach for printing PS- $\mu$ bead pixels for biochip applications. The transfer experiments were carried out onto Thermanox coverslip substrates and optical microscopy images reveal that pixels with clearly cut edges can be obtained for laser transfer fluence of higher than $0.7 \mathrm{~J} / \mathrm{cm}^{2}$ using $308 \mathrm{~nm}$ irradiation and a 100 $\mathrm{nm}$ thick triazene polymer film as DRL. The transferred pixels are very adherent to the surface of the substrates, therefore no limitation are expected for subsequent experiments in

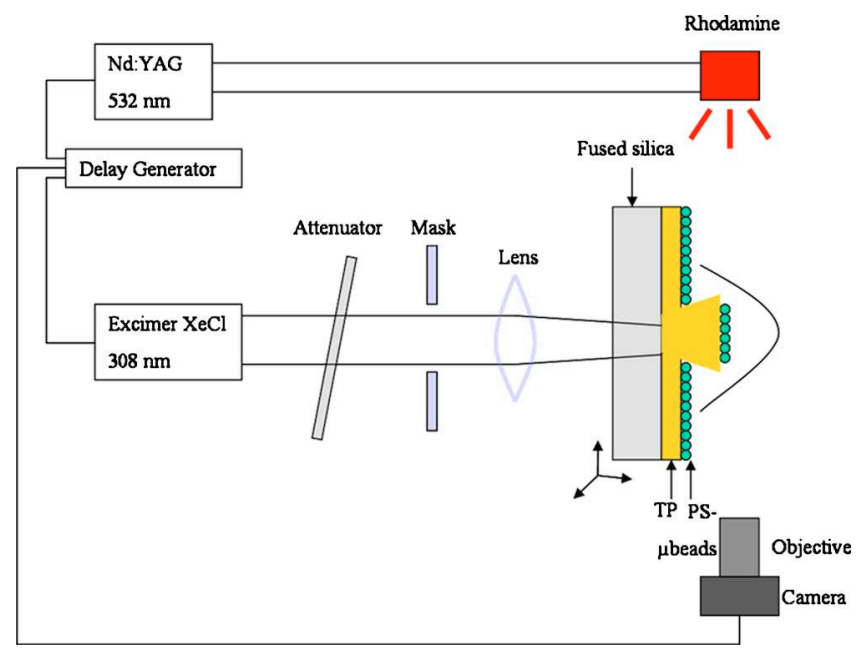

FIG. 7. (Color online) Scheme of the pump probe setup used to record the shadowgraphy images. 


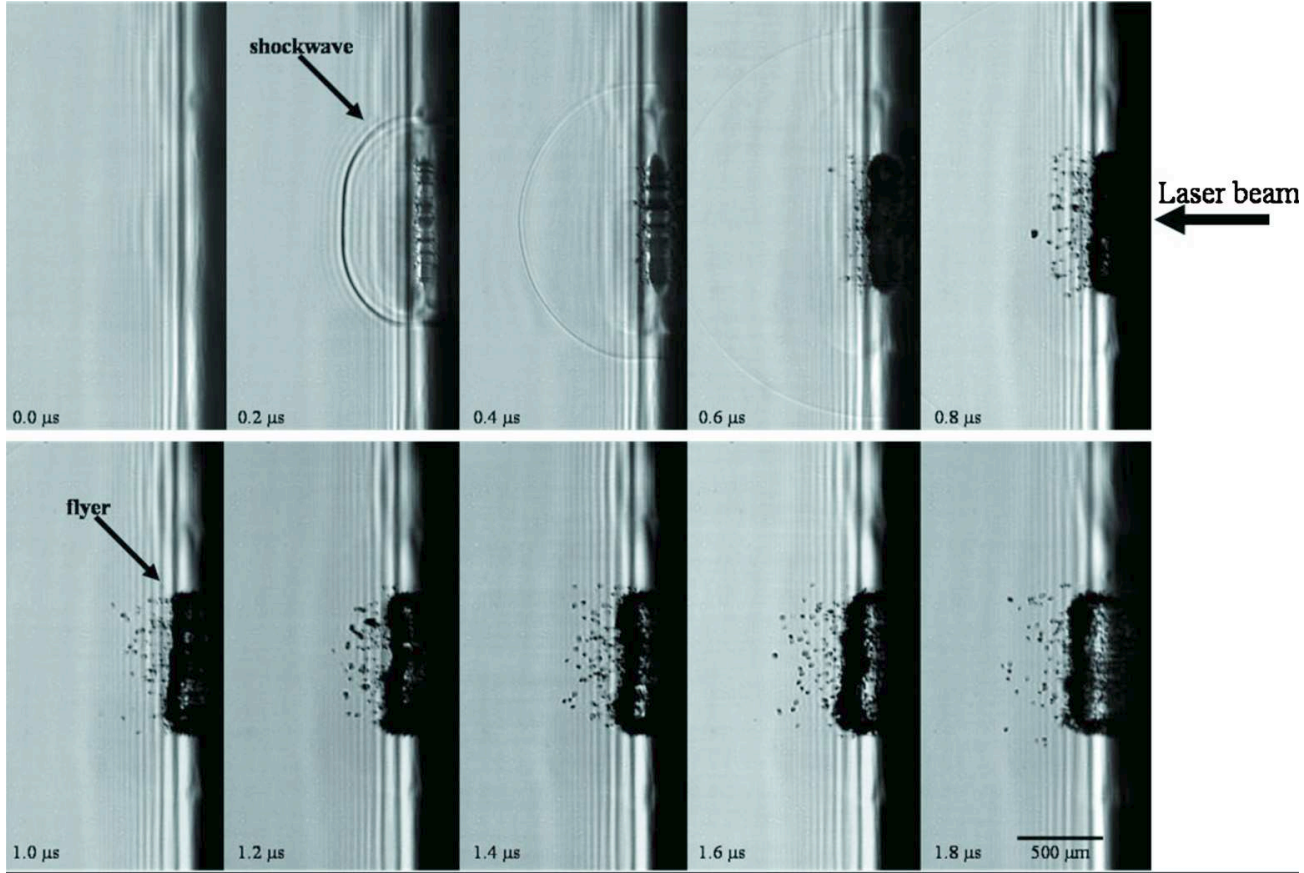

FIG. 8. (Color online) Sequence of pictures taken for back side ablation of a PS- $\mu$ bead monolayer on top of a $100 \mathrm{~nm}$ TP layer at $1.5 \mathrm{~J} / \mathrm{cm}^{2}$. The time delays are indicated in the images.

various environments. Raman spectroscopy data suggest that the chemical composition of the polystyrene beads does not change for all laser fluences applied for the transfer.

The LIFT of PS- $\mu$ beads was studied by time resolved shadowgraphy. The PS bead pixels are most probably transferred as intact layer and different sizes of pixels can be obtained by adjusting the laser parameter. The fact that these arrays can be produced cost-effective and time-effective with a minimum sample volume required suggests that LIFT is a promising technique for transferring PS bead pixels, which can therefore be applied for an increasing amount of applications.

\section{ACKNOWLEDGMENTS}

The authors would like to thank M. Nagel for synthesizing the triazene polymer. Financial support from the NATO under Project No. SfP 982671 and National Project IDEI No. $1197 / 2008$ are gratefully acknowledged.

${ }^{1}$ K. K. Jain, Curr. Opin. Drug Discovery Dev. 7, 285 (2004).

${ }^{2}$ A. Y. Rubina, A. Kolchinsky, A. A. Makarov, and A. S. Zasedatelev, Proteomics 8, 817 (2008).

${ }^{3}$ G. Hardiman, Methods Mol. Biol. 448, 21 (2008).

${ }^{4}$ B. Behkam and M. Sitti, 7th IEEE Conference on Nanotechnology, 2-5 August 2007, Hong-Kong, China, pp. 723-727.

${ }^{5}$ M. H. Lu and Y. Zhang, Adv. Mater. 18, 3094 (2006).

${ }^{6}$ C. T. Lim and Y. Zhang, Small 3, 714 (2007).

${ }^{7}$ G. Medoro, P. Vulto, L. Altomare, M. Abonnenc, A. Romani, M. Tartagni,

R. Guerrieri, and N. Manaresi, Proc. IEEE 1, 76 (2004).

${ }^{8}$ Q. Xu and K. S. Lam, J. Biomed. Biotechnol. 2003257.

${ }^{9}$ M. R. Dusseiller, D. Schlaepfer, M. Koch, R. Kroschewski, and M. Textor, Biomaterials 26, 5917 (2005).

${ }^{10}$ L. Malaquin, T. Kraus, H. Schmid, E. Delamarche, and H. Wolf, Langmuir 23, 11513 (2007)

${ }^{11}$ P. Serra, J. M. Fernandez-Pradas, F. X. Berthet, M. Colina, J. Elvira, and J. L. Morenza, Appl. Phys. A: Mater. Sci. Process. 79, 949 (2004).

${ }^{12}$ I. Zergioti, S. Mailis, N. A. Vainos, P. Papakonstantinou, C. Kalpouzos, C.
P. Grigoropoulous, and C. Fotakis, Appl. Phys. A: Mater. Sci. Process. 66, 579 (1998).

${ }^{13}$ A. Klini, A. Mourka, V. Dinca, C. Fotakis, and F. Claeyssens, Appl. Phys. A: Mater. Sci. Process. 87, 17 (2007).

${ }^{14}$ T. V. Kononenko, P. Alloncle, V. I. Konov, and M. Sentis, Appl. Phys. A: Mater. Sci. Process. 94, 531 (2009)

${ }^{15}$ J. M. Fernandez-Pradas, M. Colina, P. Serra, J. Domınguez, and J. L. Morenza, Thin Solid Films 27, 453 (2004).

${ }^{16}$ V. Dinca, A. Ranella, M. Farsari, D. Kafetzopoulous, M. Dinescu, A. Popescu, and C. Fotakis, Biomed. Microdevices 10, 719 (2008).

${ }^{17}$ A. Karaiskou, I. Zergioti, C. Fotakis, M. Kapsetaki, and D. Kafetzopoulos, Appl. Surf. Sci. 245, 208 (2003).

${ }^{18}$ P. Serra, M. Colina, J. M. Fernandez-Paras, L. Sevilla, and J. L. Morenza, Appl. Phys. Lett. 85, 1639 (2004).

${ }^{19}$ B. R. Ringeisen, D. B. Chrisey, A. Piqué, D. Krizman, M. Brooks, B. Spargo, R. C. Y. Auyeung, and P. K. Wu, Am. Biotechnol. Lab 19, 42 (2001).

${ }^{20}$ A. Doraiswamy, R. J. Narayan, T. Lippert, L. Urech, A. Wokaun, M. Nagel, B. Hopp, M. Dinescu, R. Modi, R. C. Y. Auyeung, and D. B. Chrisey, Appl. Surf. Sci. 252, 4743 (2006).

${ }^{21}$ D. B. Chrisey, A. Pique, R. A. McGill, J. S. Horwitz, B. R. Ringeisen, D. M. Bubb, and P. K. Wu, Chem. Rev. 103, 553 (2003).

${ }^{22}$ R. Fardel, M. Nagel, F. Nuesch, T. K. Lippert, and A. Wokaun, Appl. Surf. Sci. 254, 1322 (2007).

${ }^{23}$ M. Nagel, R. Fardel, P. Feurer, M. Häberli, F. Nüesch, T. K. Lippert, and A. Wokaun, Appl. Phys. A: Mater. Sci. Process. 92, 781 (2008).

${ }^{24}$ M. Nagel, R. Hany, T. Lippert, M. Molberg, F. A. Nüesch, and D. Rentsch, Macromol. Chem. Phys. 208, 277 (2007).

${ }^{25}$ T. Lippert, L. S. Bennett, T. Nakamura, H. Niino, A. Ouchi, and A. Yabe, Appl. Phys. A: Mater. Sci. Process. 63, 257 (1996).

${ }^{26}$ T. Lippert, Adv. Polym. Sci. 168, 51 (2004).

${ }^{27}$ T. Lippert, Plasma Processes Polym. 2, 525 (2005).

${ }^{28}$ R. Fardel, M. Nagel, F. Nuesch, T. Lippert, and A. Wokaun, Appl. Surf. Sci. 255, 5430 (2009)

${ }^{29}$ C. Y. Liang and S. Krimm, J. Polym. Sci., Polym. Phys. Ed. XXVI, 241 (1958).

${ }^{30}$ J. Lee, O. Kim, J. Jung, K. Na, P. Heo, and J. Hyun, Colloids Surf., B 72, 173 (2009).

${ }^{31}$ R. Fardel, M. Nagel, F. Nüesch, T. Lippert, and A. Wokaun, J. Phys. Chem. C 113, 11628 (2009).

${ }^{32}$ H. Andersson, C. Jonsson, C. Moberg, and G. Stemme, Talanta 56, 301 (2002). 